\title{
Consistent Atomic Geometries and Electronic Structure of Five Phases of Potassium Niobate from Density-Functional Theory
}

\author{
Falko Schmidt, ${ }^{1}$ Marc Landmann, ${ }^{1}$ Eva Rauls, ${ }^{1}$ Nicola Argiolas, ${ }^{2}$ Simone Sanna, \\ W. G. Schmidt, ${ }^{1}$ and Arno Schindlmayr ${ }^{1}$ \\ ${ }^{1}$ Department Physik, Universität Paderborn, 33095 Paderborn, Germany \\ ${ }^{2}$ Dipartimento di Fisica e Astronomia, Universitá di Padova, 35131 Padova, Italy
}

Correspondence should be addressed to Arno Schindlmayr; arno.schindlmayr@uni-paderborn.de

Received 8 September 2016; Revised 11 November 2016; Accepted 24 November 2016; Published 30 January 2017

Academic Editor: Pascal Roussel

Copyright (C) 2017 Falko Schmidt et al. This is an open access article distributed under the Creative Commons Attribution License, which permits unrestricted use, distribution, and reproduction in any medium, provided the original work is properly cited.

We perform a comprehensive theoretical study of the structural and electronic properties of potassium niobate $\left(\mathrm{KNbO}_{3}\right)$ in the cubic, tetragonal, orthorhombic, monoclinic, and rhombohedral phase, based on density-functional theory. The influence of different parametrizations of the exchange-correlation functional on the investigated properties is analyzed in detail, and the results are compared to available experimental data. We argue that the PBEsol and AM05 generalized gradient approximations as well as the RTPSS meta-generalized gradient approximation yield consistently accurate structural data for both the external and internal degrees of freedom and are overall superior to the local-density approximation or other conventional generalized gradient approximations for the structural characterization of $\mathrm{KNbO}_{3}$. Band-structure calculations using a HSE-type hybrid functional further indicate significant near degeneracies of band-edge states in all phases which are expected to be relevant for the optical response of the material.

\section{Introduction}

The study of perovskites, oxidic compounds with the chemical formula $\mathrm{ABO}_{3}$ and an ideal or distorted cubic crystal structure, is of both academic and technological interest. On one hand, perovskite minerals are the most abundant components in the Earth's mantle [1]. On the other hand, they exhibit a large variety of interesting physical properties $[2,3]$, which can be modified by composition-induced structural modifications.

Among the human-made perovskites, potassium niobate $\left(\mathrm{KNbO}_{3}\right)$ belongs to the important class of perovskitestructure ferroelectrics [4]. It crystallizes at about $1325 \mathrm{~K}$ in a paraelectric cubic phase (space group $\operatorname{Pm} \overline{3} m$ ) and, similar to $\mathrm{BaTiO}_{3}$ [5], undergoes three successive ferroelectric phase transitions [6]: to a tetragonal phase (space group $P 4 \mathrm{~mm}$ ) at $691 \mathrm{~K}$, from the tetragonal to an orthorhombic phase (space group Amm2) at $498 \mathrm{~K}$, and from the orthorhombic to a rhombohedral phase (space group $R 3 m$ ) at $263 \mathrm{~K}$. In addition, a new monoclinic phase (space group $P m$ ) was very recently discovered in $\mathrm{KNbO}_{3}$ nanomaterials synthesized via a hydrothermal method [7]. It has long been known that the room-temperature orthorhombic phase, in particular, is characterized by unusual dielectric properties and an exceptionally large nonlinear optical coefficient. For this reason, $\mathrm{KNbO}_{3}$ is widely used in nonlinear optics and holographic applications, where it represents a viable alternative to the more diffused $\mathrm{LiNbO}_{3}$.

Due to its technological importance, pure and defective $\mathrm{KNbO}_{3}$ has been subject to many experimental [812] and theoretical [13-19] studies. The previous theoretical investigations were typically aimed at the description of the ground-state structure and the identification of defect centers relevant for the optical properties. For the most part, they used density-functional theory $[20,21]$ with classical local or semilocal exchange-correlation functionals or even simpler semiempirical models. While these approaches are appropriate for atomic geometries and the mechanical properties of a material if not too high accuracy is required, they are much less accurate with regard to the electronic and 
optical properties, because important factors like quasiparticle renormalization of the electronic band gap, thermal effects due to the electron-phonon coupling, or excitonic effects in the linear and nonlinear optical absorption are missing. Furthermore, most of the published work so far concentrated on the structurally simple cubic and tetragonal phases, while less attention has been given to the remaining polymorphs. As a consequence, a thorough quantitative $a b$ initio study of the electronic and optical properties of $\mathrm{KNbO}_{3}$, especially of the more complex but technologically relevant room-temperature phase, with state-of-the-art methods that go beyond density-functional theory and take the abovementioned effects into account, as recently carried out for its sister material $\mathrm{LiNbO}_{3}[22-25]$, is still missing. The accurate theoretical description of the atomic structure of the relevant $\mathrm{KNbO}_{3}$ phases remains a prerequisite for all such studies, however.

In order to pave the way for future investigations along these lines, here we perform a comprehensive study of the structural and electronic properties of all the five known phases of $\mathrm{KNbO}_{3}$, including the newly discovered monoclinic phase, based on density-functional theory. The influence of different parametrizations of the exchange-correlation functional on the investigated properties is discussed in detail, and the results are compared to available experimental data. Such a systematic approach is especially important for $\mathrm{KNbO}_{3}$ because the energetic separation and the structural differences between the various phases are small and therefore potentially sensitive to details of the computational procedure. Indeed, our results show that several recently introduced exchange-correlation functionals give, overall, a better description of the atomic geometries throughout the various phases than common standard functionals as used in earlier studies. Band-structure calculations are additionally performed with a nonlocal hybrid functional to correct the well-known underestimation of the fundamental band gap incurred with conventional local or semilocal functionals, which stems from the absence of a derivative discontinuity with respect to the particle number as in the exact functional $[26,27]$.

This paper is organized as follows. In Section 2, we give an overview of our computational method. In Section 3, we present our results for the optimized atomic geometries of the five considered phases of $\mathrm{KNbO}_{3}$ and compare the performances of different exchange-correlation functionals in relation to experimental data. Furthermore, we discuss the effects of the differently broken symmetries on the electronic band structure through the successive phase transitions. We finally summarize our conclusions in Section 4.

\section{Computational Method}

All calculations of this study are performed with the Vienna $A b$ initio Simulation Package (VASP) [28, 29], a planewave implementation of density-functional theory (DFT). The electron-ion interaction is described by the projectoraugmented-wave (PAW) scheme $[29,30]$, where the K $3 s$ and the $\mathrm{Nb} 4 s$ electrons are treated explicitly as valence states. For the exchange and correlation between the electrons, we test several different schemes in order to compare their performance, namely, the local-density approximation (LDA), semilocal generalized gradient approximations (GGA), a semilocal meta-GGA, and a nonlocal hybrid functional. In detail, we use the LDA as parametrized by Perdew and Zunger [31]. The conventional Perdew-Burke-Ernzerhof (PBE) parametrization [32] and the more recently introduced PBEsol [33] and AM05 [34] functionals are considered on the GGA side; the latter two are chosen because they are known to yield accurate lattice parameters comparable to hybridfunctional DFT calculations at a moderate computational cost [35]. Furthermore, the revised Tao-Perdew-StaroverovScuseria (RTPSS) meta-GGA [36] is employed. Bandstructure calculations are additionally performed for the relaxed PBEsol geometries with the Heyd-Scuseria-Ernzerhof (HSE) screened-Coulomb-potential hybrid functional $[37,38]$. The fraction of the exact exchange from HartreeFock theory, 25\% in the conventional HSE06 functional, is increased to $30 \%$ to match the experimentally observed band gap of the cubic $\mathrm{KNbO}_{3}$ phase. Nonlocal HSE-type hybrid functionals offer a reliable way to approximate more rigorous band-structure calculation schemes within the framework of quasiparticle theory and thus effectively overcome the band gap problem of conventional DFT calculations with local or semilocal exchange-correlation functionals at a lower numerical cost $[39,40]$.

The electronic wave functions are expanded into plane waves up to a kinetic energy of $600 \mathrm{eV}$ for the lattice-parameter optimization. All structural degrees of freedom are relaxed until the forces on each atom are below $0.001 \mathrm{meV} / \AA$. The cutoff energy is reduced to $400 \mathrm{eV}$ in the hybrid-functional band-structure calculations. The Brillouin-zone integrations are performed using a shifted Monkhorst-Pack $6 \times 6$ $\times 6 \mathrm{k}$-point mesh for all $\mathrm{KNbO}_{3}$ phases. In calculations with the HSE hybrid functional, a regular $\Gamma$-centered $6 \times 6 \times 6 \mathbf{k}$ point mesh is used instead.

\section{Results and Discussion}

Figure 1 illustrates the five different phases of $\mathrm{KNbO}_{3}$ considered here with exaggerated deformations from the cubic configuration (Figure 1(a)). Starting from the cubic phase, the tetragonal phase is reached by elongation along $a$ axis and compression along the other two axes (Figure 1(b)). Further distortions of the lattice parameters transform the tetragonal phase into an orthorhombic polymorph with distinct lattice parameters $a^{\prime}, b^{\prime}$, and $c^{\prime}$ (Figure 1(c), left). The crystal structure of orthorhombic $\mathrm{KNbO}_{3}$, whose rectangular unit cell contains two formula units, that is, ten atoms, can alternatively be characterized by a smaller rhombic unit cell with lattice parameters $a \neq b=c$ and $\alpha \neq \beta=\gamma=90^{\circ}$ which contains only one formula unit (Figure 1(c), right); it is this nonrectangular primitive unit cell that we employ in all structure optimizations and electronic-structure calculations. The relation between the orthorhombic and the rhombic unit cells, including the ion displacements, is further illustrated in Figure 2. The orthorhombic phase transforms into the monoclinic phase by choosing mutually distinct lattice parameters $a \neq b \neq c$ (Figure 1(d)). Alternatively, 


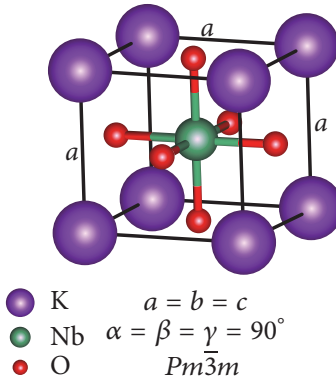

(a)

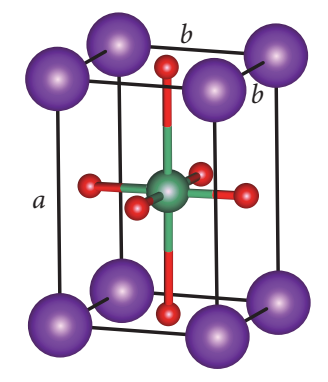

$$
\begin{array}{llc} 
& \mathrm{K} & a \neq b=c \\
& \mathrm{Nb} & \alpha=\beta=\gamma=90^{\circ} \\
& \mathrm{O} & \text { P4mm }
\end{array}
$$

$P 4 \mathrm{~mm}$

(b)
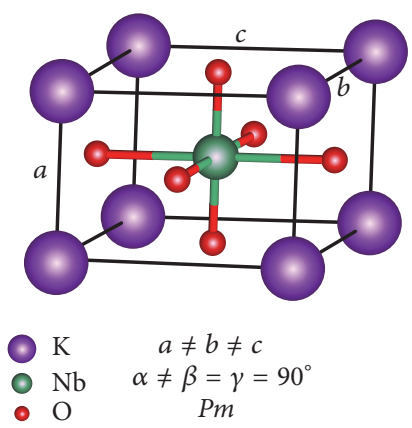

(d)

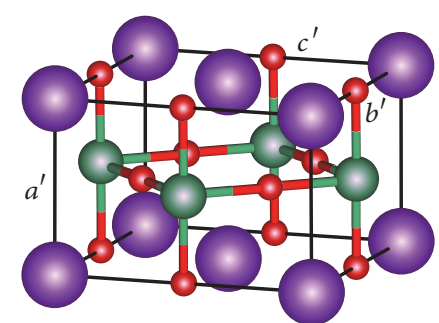
K $\quad a^{\prime} \neq b^{\prime} \neq c$
- $\mathrm{Nb} \quad \alpha^{\prime}=\beta^{\prime}=\gamma^{\prime}=90$
- $\mathrm{O} A m m 2$

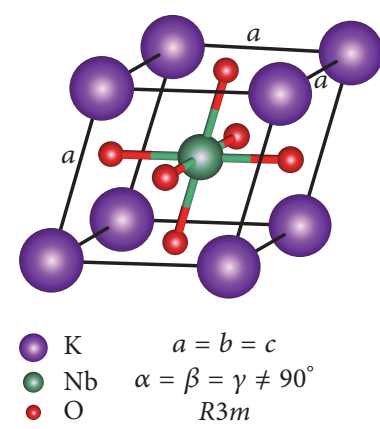

(e)

(c)

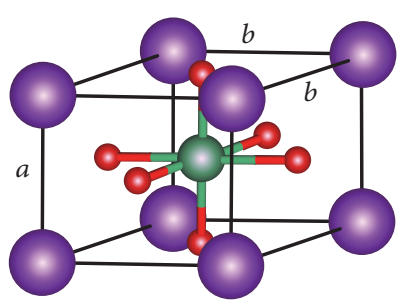

$a \neq b=c$

$\alpha \neq \beta=\gamma=90^{\circ}$

Figure 1: Different phases of $\mathrm{KNbO}_{3}$ : (a) cubic, (b) tetragonal, (c) orthorhombic (left: conventional orthorhombic unit cell, right: primitive rhombic unit cell), (d) monoclinic, and (e) rhombohedral with lattice parameters, angles, and space groups in Hermann-Mauguin notation. The deformations are actually small and are exaggerated here for clarity.

the rhombohedral phase is obtained by choosing equal lattice parameters $a=b=c$ together with equal but acute angles $\alpha=\beta=\gamma$ (Figure 1(e)).

3.1. Structure Optimization. In order to study the influence of different approximations for exchange and correlation on the DFT results for $\mathrm{KNbO}_{3}$, we perform complete structure optimizations of the lattice parameters and the atomic displacements for all five phases using the LDA, PBE, PBEsol, AM05, and RTPSS functionals. We start with the hightemperature cubic phase and then follow the order in which the phases would occur for decreasing temperature.

Table 1 lists the calculated lattice parameter $a$ and the optimized unit-cell volume $V$ for the cubic phase obtained with the different exchange-correlation functionals as well as experimental data for comparison. Consistent with the cubic symmetry, all lattice parameters have precisely the same value, and all lattice vectors are perpendicular to each other. There are no internal degrees of freedom and hence no atomic displacements in the cubic phase. At first glance, the PBE results appear to agree best with the experimental lattice constant, but it must be borne in mind that the measurement was performed at $708 \mathrm{~K}$, whereas the calculations correspond to $0 \mathrm{~K}$. The good quantitative agreement is in fact fortuitous and due to an error cancellation between the neglect of thermal expansion and the well-known tendency of PBE (and other conventional GGAs) to overestimate lattice constants

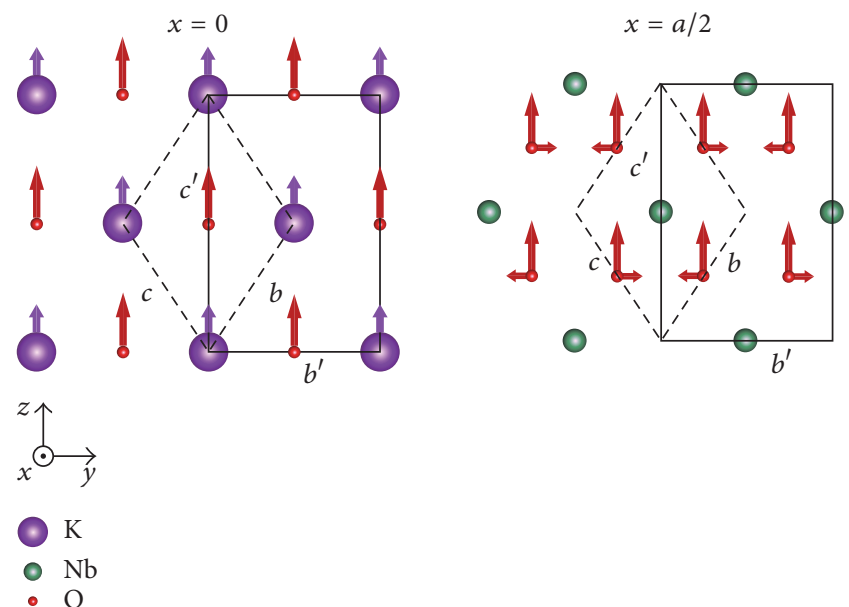

FIgURE 2: Atomic displacements in the conventional orthorhombic (solid lines) and primitive rhombic (dashed lines) unit cells of the orthorhombic $\mathrm{KNbO}_{3}$ polymorph. The unit cell is split into two layers of atoms perpendicular to $a$ axis. The deformations are exaggerated for clarity.

at zero temperature. The LDA predicts the smallest value for $a$, while the results from other functionals are in between; the same applies to the equilibrium unit-cell volume. In concordance with literature data on various other materials, 
TABLE 1: Lattice parameter $a$ and equilibrium unit-cell volume $V$ calculated with different exchange-correlation functionals for the cubic phase of $\mathrm{KNbO}_{3}$. Data in brackets represent relative deviations from the experimental values in percent.

\begin{tabular}{|c|c|c|c|c|c|c|}
\hline$P m \overline{3} m$ & LDA & PBE & PBEsol & AM05 & RTPSS & Expt. $^{a}$ \\
\hline$a(\AA)$ & $3.954(-1.69)$ & $4.024(+0.05)$ & $3.985(-0.92)$ & $3.989(-0.82)$ & $4.008(-0.35)$ & 4.022 \\
\hline$V\left(\AA^{3}\right)$ & $61.72(-5.13)$ & $65.16(+0.15)$ & $63.28(-2.74)$ & $63.47(-2.44)$ & $64.38(-1.05)$ & 65.06 \\
\hline
\end{tabular}

${ }^{\mathrm{a}}$ Ref. [41]; measured at $708 \mathrm{~K}$.

TABLE 2: Lattice parameters $a$ and $b$, ratio $a / b$, equilibrium unit-cell volume $V$, atomic displacements along $a$ axis in percent of $a$, and total-energy difference $\Delta E$ per formula unit with respect to cubic $\mathrm{KNbO}_{3}$ calculated with different exchange-correlation functionals for the tetragonal phase of $\mathrm{KNbO}_{3}$. Data in brackets represent relative deviations from the experimental values in percent.

\begin{tabular}{|c|c|c|c|c|c|c|}
\hline$P 4 m m$ & LDA & PBE & PBEsol & AM05 & RTPSS & Expt. $^{a}$ \\
\hline$a(\AA)$ & $3.989(-1.82)$ & $4.193(+3.20)$ & $4.058(-0.12)$ & $4.098(+0.86)$ & $4.085(+0.54)$ & 4.063 \\
\hline$b(\AA)$ & $3.945(-1.30)$ & $3.994(-0.08)$ & $3.969(-0.70)$ & $3.968(-0.73)$ & $3.992(-0.13)$ & 3.997 \\
\hline$a / b$ & $1.0112(-0.52)$ & $1.0498(+3.28)$ & $1.0224(+0.58)$ & $1.0328(+1.60)$ & $1.0233(+0.67)$ & 1.0165 \\
\hline$V\left(\AA^{3}\right)$ & $62.08(-4.36)$ & $66.87(+3.02)$ & $63.93(-1.51)$ & $64.52(-0.60)$ & $65.10(+0.29)$ & 64.91 \\
\hline$\Delta \mathrm{K}$ & 1.072 & 1.762 & 1.462 & 1.635 & 1.634 & 1.8 \\
\hline$\Delta \mathrm{Nb}$ & 0 & 0 & 0 & 0 & 0 & 0 \\
\hline$\Delta \mathrm{O} 1$ & 2.859 & 6.142 & 4.109 & 4.972 & 4.277 & 4.4 \\
\hline$\Delta \mathrm{O} 2$ & 3.026 & 4.962 & 3.850 & 4.352 & 3.934 & 4.0 \\
\hline$\Delta \mathrm{O} 3$ & 3.026 & 4.962 & 3.850 & 4.352 & 3.934 & 4.0 \\
\hline$\Delta E(\mathrm{eV})$ & -0.0069 & -0.0522 & -0.0201 & -0.0330 & -0.0231 & \\
\hline
\end{tabular}

${ }^{\mathrm{a}}$ Ref. [41]; measured at $543 \mathrm{~K}$.

we find that PBEsol, AM05, and RTPSS provide accurate and quite similar lattice parameters [36, 42-46].

For the tetragonal phase, the data listed in Table 2 is obtained with equal lattice parameters $b$ and $c$ and perpendicular lattice vectors. In contrast to the cubic phase, the displacements do not vanish in this case. If we keep the $\mathrm{Nb}$ atom fixed at its ideal position in the center of the unit cell, we observe that all other atoms shift towards positive values along $a$ axis; displacements along the other axes are forbidden due to symmetry constraints. Since the experimental lattice parameters shown for comparison refer to $543 \mathrm{~K}$ and include effects of thermal expansion, the ratio $a / b$ is also listed. The lattice parameter $a$ is best matched by PBEsol, while the PBE value for $b$ is closest to the experiment. The latter is fortuitous, however, as indicated by the large overestimation of $a$ which exceeds 3\% and constitutes the largest deviation among all tested functionals. The deficiency of the PBE functional is further reflected in the ratio $a / b$ of 1.0498 , which shows the strongest deviation from the experimental value of 1.0165 , while LDA (1.0112) slightly underestimates the experimental value, PBEsol (1.0224) and RTPSS (1.0233) slightly overestimate it by a similar amount, and AM05 (1.0328) yields a somewhat larger ratio. Despite the reasonably accurate LDA lattice-parameter ratio, the associated equilibrium volume of the unit cell of $62.08 \AA^{3}$ shows the poorest agreement with the experimental volume of $64.91 \AA^{3}$. The equilibrium volume is excellently reproduced by the RTPSS value of $65.10 \AA^{3}$, followed by the AM05 value of $64.52 \AA$. As for the relative atomic displacements, all functionals except the LDA, which predicts systematically too small shifts, are in reasonable agreement with the experimental data. However, RTPSS clearly shows the best performance in the description of these internal degrees of freedom, aside from the accidentally close PBE displacement of the $\mathrm{K}$ atom. Overall, we thus conclude that PBEsol and RTPSS are well suited to describe the tetragonal phase of $\mathrm{KNbO}_{3}$, although RTPSS provides the most balanced description of all structural degrees of freedom. This highlights the importance of incorporating all external as well as internal degrees of freedom in the evaluation of functional performance for structural properties.

Table 3 contains our results for the orthorhombic phase. We point out again that the primitive rhombic unit cell is not rectangular, as only the double unit cell exhibits the actual orthorhombic structure. The arrows in Figure 2 indicate the direction of the displacements in the plane perpendicular to $a$ axis; there are no detectable displacements parallel to $a$. As can be seen, the atoms shift mainly along $c^{\prime}$ direction of the double unit cell. In the rotated basis system of the primitive single unit cell, the displacements have roughly equal components along $b$ and $c$ directions. As before, the LDA lattice parameters and volume substantially underestimate the experimental data. In addition, LDA tends strongly towards cubic symmetry, as indicated by the almost right angle of $89.91^{\circ}, a / b$ ratio of 0.9922 that is close to unity, and the significantly underestimated atomic displacements. The PBE parametrization shows the smallest (for $a$ ) and largest (for $b$ ) deviations from the experimental values among all considered functionals, indicating a similar imbalance in the description of lattice parameters as observed for the tetragonal phase. In contrast, the functionals PBEsol, AM05, and RTPSS provide reasonable lattice parameters with comparably small deviations from the experimental values for both $a$ and $b$. This more balanced description of lattice parameters 
TABLE 3: Lattice parameters $a$ and $b$, angle $\alpha$, ratio $a / b$, equilibrium unit-cell volume $V$, atomic displacements in percent of $b$, and total-energy difference $\Delta E$ per formula unit with respect to cubic $\mathrm{KNbO}_{3}$ calculated with different exchange-correlation functionals for the orthorhombic phase of $\mathrm{KNbO}_{3}$. Data in brackets represent relative deviations from the experimental values in percent.

\begin{tabular}{lcccccc}
\hline$A m m 2$ & LDA & PBE & PBEsol & AM05 & RTPSS $^{\text {Expt. }^{a}}$ \\
\hline$a(\AA)$ & $3.940(-0.83)$ & $3.983(+0.25)$ & $3.961(-0.30)$ & $3.958(-0.38)$ & $3.984(+0.28)$ & 3.973 \\
$b(\AA)$ & $3.971(-1.59)$ & $4.100(+1.61)$ & $4.021(-0.35)$ & $4.043(+0.20)$ & $4.048(+0.32)$ & 4.035 \\
$\alpha\left(^{\circ}\right)$ & $89.91(+0.20)$ & $89.66(-0.08)$ & $89.82(+0.10)$ & $89.76(+0.03)$ & $89.81(+0.09)$ & 89.73 \\
$a / b$ & $0.9922(+0.77)$ & $0.9715(-1.33)$ & $0.9851(+0.05)$ & $0.9790(-0.57)$ & $0.9842(-0.04)$ & 0.9846 \\
$V\left(\AA^{3}\right)$ & $62.13(-3.94)$ & $66.95(+3.51)$ & $64.04(-0.99)$ & $64.70(+0.03)$ & $65.28(+0.93)$ & 64.68 \\
$\Delta \mathrm{K}$ & 0.632 & 1.095 & 0.877 & 0.987 & 0.971 & 0 \\
$\Delta \mathrm{Nb}$ & 0 & 0 & 0 & 2.618 & 0 \\
$\Delta \mathrm{O} 1$ & 1.793 & 2.942 & 2.306 & 2.432 & 2.381 \\
$\Delta \mathrm{O} 2$ & 1.615 & 2.768 & 2.131 & 2.432 & 2.216 \\
$\Delta \mathrm{O} 3$ & 1.615 & 2.768 & 2.131 & -0.0401 & 2.568 \\
$\Delta E(\mathrm{eV})$ & -0.0080 & -0.0617 & -0.0245 & 2.416 & 2.413 \\
\hline $\mathrm{Ref}$ & & & & & -0.0289 \\
\hline
\end{tabular}

${ }^{a}$ Ref. [41]; measured at $295 \mathrm{~K}$.

is underlined by improved $a / b$ ratios with PBEsol (0.9851) and RTPSS (0.9842) which show virtually identical but opposing minimal deviations from the experimental value (0.9846). While all functionals predict a very similar small reduction of angle $\alpha$, the experimental value of $89.73^{\circ}$ is most accurately reproduced by AM05 $\left(89.76^{\circ}\right)$. AM05 also yields the equilibrium volume (64.70) closest to experiment (64.68). The three functionals PBEsol, AM05, and RTPSS not only yield results in reasonable quantitative agreement with the experimental data but also give a good account of the atomic displacements, although the best description of the internal degrees of freedom is provided by the AM05 parametrization. Overall, AM05 provides the best balanced description of all structural degrees of freedom in orthorhombic $\mathrm{KNbO}_{3}$.

The results for the newly discovered monoclinic phase depend sensitively on the starting parameters for the atomic displacements, as the structure tends to relax to the orthorhombic phase for poorly chosen initial displacements. The only previous theoretical study of $\mathrm{KNbO}_{3}$ in the monoclinic phase was reported by Zhang et al. [48]. In our simulations, we take the displacements given in [48] as the initial configuration. In this way, we eventually obtain a properly relaxed monoclinic structure, which corresponds to a local minimum of the total-energy landscape, indicating that the monoclinic phase is metastable not only for $\mathrm{KNbO}_{3}$ nanomaterials but also for the bulk. The results listed in Table 4 show that the performance of the different functionals is the same as that for the related orthorhombic phase, which occurs in the same temperature range. The universal trends of underestimation and overestimation of lattice parameters by the LDA and PBE functionals are clearly reflected in equilibrium volumes of $62.13 \AA^{3}$ and $66.99 \AA^{3}$ which fall below or exceed the experimental volume of $64.86 \AA^{3}$, respectively. Surprisingly, LDA performs best for angle $\alpha$ as well as for the ratios $a / b$ and $a / c$. A notable improvement over the LDA and PBE values is observable for the other functionals, but no clear trend emerges. PBEsol, AM05, and RTPSS provide virtually the same accuracy for the lattice parameters. The atomic displacements have again roughly equal components along $b$ and $c$ directions and no detectable component along a. Compared to PBEsol and RTPSS, AM05 shows a slight tendency towards larger atomic displacements. Although there are no directly comparable experimental values, the calculated displacements are compatible with those of Zhang et al. [48] based on density-functional theory in combination with the otherwise rarely used Wu-Cohen functional [49].

Finally, for the rhombohedral phase, we set the starting values for the displacements of all atoms except $\mathrm{Nb}$ to two percent along each lattice vector, because the structure tends to relax to the cubic phase for too small initial displacements. The resulting optimized values are listed in Table 5. The lattice parameter $a$ and angle $\alpha$ obtained from PBEsol, AM05, and RTPSS are overall in good agreement with the experimental data. The performance of LDA and PBE follows that for the other phases and shows characteristic underestimation and overestimation of the lattice parameters, respectively. Our calculated atomic displacements are notably larger than those reported by Fontana et al. in 1984 [41], especially for the oxygen atoms. The reason for this discrepancy remains unclear. On one hand, the theoretical results are very consistent for all functionals considered here and thus appear robust. On the other hand, the low-temperature rhombohedral phase has received relatively little attention in the literature so far, so that there are no other, more recent experimental characterizations of this structure which could be used for further comparison. In our simulations, all displacements are predominantly along the spatial diagonal of the unit cell with roughly equal components along all three lattice vectors.

3.2. Phase Stability. For the purpose of comparison, Table 6 summarizes our results for the total-energy differences $\Delta E$ per formula unit for the various phases of $\mathrm{KNbO}_{3}$, which correspond to the formation energies relative to the cubic polymorph. Although the numerical differences are generally small, we find that the rhombohedral structure is energetically favored by all exchange-correlation functionals considered in this work. As the calculations refer to $0 \mathrm{~K}$, this is gratifyingly consistent with the experimental fact that the 
TABLE 4: Lattice parameters $a, b$, and $c$, angle $\alpha$, ratios $a / b$ and $a / c$, equilibrium unit-cell volume $V$, atomic displacements in percent of $b$, and total-energy difference $\Delta E$ per formula unit with respect to cubic $\mathrm{KNbO}_{3}$ calculated with different exchange-correlation functionals for the monoclinic phase of $\mathrm{KNbO}_{3}$. Data in brackets represent relative deviations from the experimental values in percent.

\begin{tabular}{|c|c|c|c|c|c|c|}
\hline$P m$ & LDA & PBE & PBEsol & AM05 & RTPSS & Expt. $^{a}$ \\
\hline$a(\AA)$ & $3.940(-1.28)$ & $3.983(-0.20)$ & $3.961(-0.75)$ & $3.958(-0.83)$ & $3.984(-0.18)$ & 3.991 \\
\hline$b(\AA)$ & $3.972(-1.83)$ & $4.104(+1.43)$ & $4.023(-0.57)$ & $4.045(-0.02)$ & $4.049(+0.07)$ & 4.046 \\
\hline$c(\AA)$ & $3.970(-1.17)$ & $4.098(+2.02)$ & $4.020(+0.07)$ & $4.041(+0.60)$ & $4.047(+0.75)$ & 4.017 \\
\hline$\alpha\left({ }^{\circ}\right)$ & $89.91(+0.02)$ & $89.66(-0.26)$ & $89.82(-0.08)$ & $89.76(-0.14)$ & $89.81(-0.09)$ & 89.89 \\
\hline$a / b$ & $0.9919(+0.56)$ & $0.9705(-1.61)$ & $0.9846(-0.18)$ & $0.9785(-0.80)$ & $0.9839(-0.25)$ & 0.9864 \\
\hline$a / c$ & $0.9924(-0.11)$ & $0.9719(-2.17)$ & $0.9853(-0.83)$ & $0.9795(-1.41)$ & $0.9844(-0.92)$ & 0.9935 \\
\hline$V\left(\AA^{3}\right)$ & $62.13(-4.21)$ & $66.99(+3.28)$ & $64.06(-1.23)$ & $64.70(-0.25)$ & $65.28(+0.65)$ & 64.86 \\
\hline$\Delta \mathrm{K}$ & 1.260 & 2.194 & 1.736 & 1.971 & 1.942 & \\
\hline$\Delta \mathrm{Nb}$ & 0 & 0 & 0 & 0 & 0 & \\
\hline$\Delta \mathrm{O} 1$ & 3.571 & 5.906 & 4.626 & 5.234 & 4.771 & \\
\hline$\Delta \mathrm{O} 2$ & 3.208 & 5.583 & 4.262 & 4.876 & 4.443 & \\
\hline$\Delta \mathrm{O} 3$ & 3.197 & 5.679 & 4.263 & 4.890 & 4.446 & \\
\hline$\Delta E(\mathrm{eV})$ & -0.0085 & -0.0623 & -0.0250 & -0.0405 & -0.0294 & \\
\hline
\end{tabular}

${ }^{\mathrm{a}}$ Ref. [47]; measured at $298 \mathrm{~K}$.

TABLE 5: Lattice parameter $a$, angle $\alpha$, equilibrium unit-cell volume $V$, atomic displacements in percent of $a$, and total-energy difference $\Delta E$ per formula unit with respect to cubic $\mathrm{KNbO}_{3}$ calculated with different exchange-correlation functionals for the rhombohedral phase of $\mathrm{KNbO}_{3}$. Data in brackets represent relative deviations from the experimental values in percent.

\begin{tabular}{|c|c|c|c|c|c|c|}
\hline$R 3 m$ & LDA & PBE & PBEsol & AM05 & RTPSS & Expt. $^{a}$ \\
\hline$a(\AA)$ & $3.961(-1.37)$ & $4.059(+1.07)$ & $4.001(-0.37)$ & $4.014(-0.05)$ & $4.027(+0.27)$ & 4.016 \\
\hline$\alpha\left({ }^{\circ}\right)$ & $89.95(+0.03)$ & $89.80(-0.13)$ & $89.90(-0.02)$ & $89.86(-0.07)$ & $89.95(+0.03)$ & 89.92 \\
\hline$V\left(\AA^{3}\right)$ & $62.15(-4.05)$ & $66.87(+3.24)$ & $64.05(-1.11)$ & $64.67(-0.15)$ & $65.30(+0.82)$ & 64.77 \\
\hline$\Delta \mathrm{K}$ & 1.296 & 2.281 & 1.794 & 2.046 & 2.037 & 1.300 \\
\hline$\Delta \mathrm{Nb}$ & 0 & 0 & 0 & 0 & 0 & 0 \\
\hline$\Delta \mathrm{O} 1$ & 3.340 & 5.548 & 4.360 & 4.945 & 4.580 & 0.301 \\
\hline$\Delta \mathrm{O} 2$ & 3.340 & 5.548 & 4.360 & 4.945 & 4.580 & 0.333 \\
\hline$\Delta \mathrm{O} 3$ & 3.340 & 5.548 & 4.360 & 4.945 & 4.580 & 0.333 \\
\hline$\Delta E(\mathrm{eV})$ & -0.0087 & -0.0623 & -0.0256 & -0.0410 & -0.0305 & \\
\hline
\end{tabular}

${ }^{\mathrm{a}}$ Ref. [41]; measured at $230 \mathrm{~K}$.

TABLE 6: Calculated total-energy differences $\Delta E$ with respect to the cubic phase in eV per formula unit with different exchangecorrelation functionals for the five phases of $\mathrm{KNbO}_{3}$.

\begin{tabular}{lccccc}
\hline Phase & LDA & PBE & PBEsol & AM05 & RTPSS \\
\hline$P m \overline{3} m$ & 0 & 0 & 0 & 0 & 0 \\
$P 4 m m$ & -0.0069 & -0.0522 & -0.0201 & -0.0330 & -0.0231 \\
$A m m 2$ & -0.0080 & -0.0617 & -0.0245 & -0.0401 & -0.0289 \\
$P m$ & -0.0085 & -0.0623 & -0.0250 & -0.0405 & -0.0294 \\
$R 3 m$ & -0.0087 & -0.0623 & -0.0256 & -0.0410 & -0.0305 \\
\hline
\end{tabular}

rhombohedral phase is stable in the low-temperature limit. It is followed, in ascending order, by the monoclinic, the orthorhombic, the tetragonal, and finally the cubic structure, consistent among all functionals. The energetic ordering of the different phases thus follows the sequence in which they become stable for increasing temperature. A proper $a b$ initio description of the phase transitions requires a numerical evaluation of the temperature-dependent free energies with the full incorporation of phonon contributions [54] which goes beyond the present study, however.

Figure 3 illustrates the correlation between the unit-cell volume and the relative formation energies. The remarkably narrow spread of the LDA results and the much larger spread of the PBE results for the different phases are clearly visible. This is in accordance with our earlier observation that the LDA tends to underestimate and PBE tends to overestimate symmetry-breaking deformations relative to the cubic structure. The PBEsol and AM05 results lie between the LDA and PBE values, indicating a more balanced description of the structure and energetics. The RTPSS results span a similar energy range as PBEsol and AM05 but are shifted to slightly larger volumes. In addition, Figure 3 shows an almost linear dependence of the formation energies on the unit-cell volume, suggesting a dominant volume effect on the phase stability of the $\mathrm{KNbO}_{3}$ polymorphs. Similar trends in the correlation between formation energies and equilibrium volume have also been observed for other oxide materials that exhibit extended structure polymorphism $[55,56]$. 


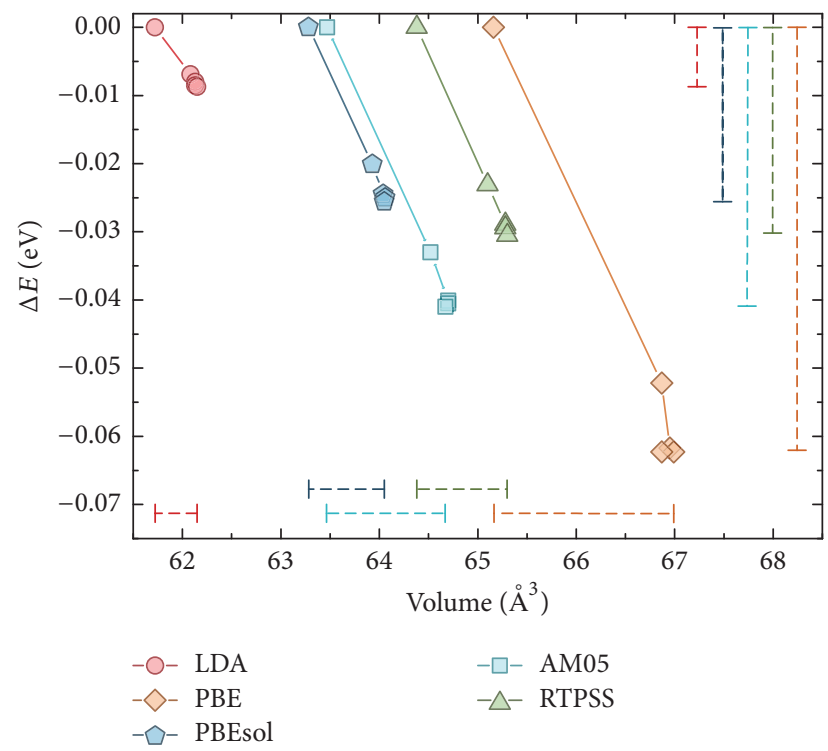

FIGURE 3: Correlation between the equilibrium unit-cell volume $V$ and the total-energy difference per formula unit $\Delta E$ with respect to the cubic phase calculated with different exchange-correlation functionals. The solid lines are intended to guide the eyes. The dashed bars indicate the total volume and energy ranges encompassing all five $\mathrm{KNbO}_{3}$ phases for a particular functional.

3.3. Functional Performance. The underestimation and overestimation of lattice parameters by LDA and by conventional generalized gradient approximations like PBE, respectively, observed consistently throughout this work for all investigated $\mathrm{KNbO}_{3}$ polymorphs, are well known and documented for a large variety of materials $[45,57,58]$. In particular, these trends were confirmed in a DFT study of various ferroelectric perovskites by Tinte et al. [59]. Nevertheless, the reasonable overall agreement with experimental data together with the well-understood performance in numerical simulations makes these established functionals a sound reference for the benchmarking of other exchange-correlation functionals. The improved prediction of structural data by PBEsol, AM05, and RTPSS observed here for $\mathrm{KNbO}_{3}$ is likewise in accordance with recent studies for simple metallic and semiconducting systems [36, 42-46, 58], but our results demonstrate that this extends to oxides with various stable and metastable polymorphs of complex symmetry, which exhibit manifold internal degrees of freedom. We note in this context that it is also possible to improve conventional semilocal functionals through restriction to specific classes of materials by introducing system-dependent parameters that modify the localization of the exchange-correlation hole [59], an idea that is not strictly ab initio but presently has a strong influence on the construction and use of hybrid functionals.

The energetic ordering (Table 6) of the five $\mathrm{KNbO}_{3}$ polymorphs is identical for all considered functionals and, most importantly, in accordance with the observed experimental sequence. Hence, even though the LDA energy differences are close to the overall numerical accuracy of current implementations of plane-wave DFT for solid-state systems,
TABLE 7: Complete list of calculated PBEsol lattice parameters for the five different phases of $\mathrm{KNbO}_{3}$, which are used in the HSE electronic-structure calculations.

\begin{tabular}{lccccc}
\hline Phase & $P m \overline{3} m$ & $P 4 m m$ & $A m m 2$ & $P m$ & $R 3 m$ \\
\hline$a(\AA)$ & 3.985 & 4.058 & 3.961 & 3.961 & 4.001 \\
$b(\AA)$ & 3.985 & 3.969 & 4.021 & 4.023 & 4.001 \\
$c(\AA)$ & 3.985 & 3.969 & 4.021 & 4.020 & 4.001 \\
$\alpha\left(^{\circ}\right)$ & 90 & 90 & 89.82 & 89.82 & 89.90 \\
$\beta\left(^{\circ}\right)$ & 90 & 90 & 90 & 90 & 89.90 \\
$\gamma\left({ }^{\circ}\right)$ & 90 & 90 & 90 & 90 & 89.90 \\
\hline
\end{tabular}

this does not disqualify any of the considered exchangecorrelation functionals from applications to $\mathrm{KNbO}_{3}$. This happenstance of correct phase ordering independent of specific functionals, especially among structurally complex oxides characterized by polymorph-rich phase diagrams, is in fact rather fortuitous. In the case of $\mathrm{TiO}_{2}$, for example, common local (LDA) and semilocal (PBE and PBEsol) as well as hybrid functionals fail to reproduce the experimentally observed energy ordering among the three main crystallographic phases rutile, anatase, and brookite, which structurally differ solely by distortions and rearrangements of the $\mathrm{TiO}_{6}$ octahedral building blocks [60-63]. Furthermore, the choice of exchange-correlation functionals in general, as well as the fraction of exact exchange in various hybrid functionals, has been found to alter the phase stability of oxide systems $[63,64]$.

The body of data for the atomic structure and phase energetics suggests that there is no good justification to stick to the LDA or to conventional GGAs when dealing with complex oxide materials. More recent parametrizations like PBEsol or AM05, which specifically aim at an accurate description of solid-state systems, as well as meta-GGAs like RTPSS, constructed to satisfy a maximum of exact constraints for the exchange-correlation functional without empirical parameters, offer a reliable and numerically efficient way to improve the accuracy of state-of-the-art ab initio simulations. All three functionals provide a sound basis for subsequent calculations. In the following, we largely restrict the analysis of the electronic structure to the PBEsol geometries summarized in Table 7, as we do not expect qualitatively different trends for geometries from AM05 or RTPSS. In view of future studies, these functionals seem to be well suited as a starting point not only for hybrid-functional DFT but also for higherlevel perturbative approaches within the framework of manybody perturbation theory [65]. The analysis of vibrational properties in $\mathrm{KNbO}_{3}$ will equally benefit from the qualitative and quantitative reliability provided by these functionals, as recently demonstrated for the related material $\mathrm{LiNbO}_{3}[24]$.

3.4. Band Structures. We now proceed to analyzing the effects of the structural deformations in the various phases on the electronic band structures. In a preliminary step, we study the influence of the structural parameters and the exchangecorrelation functionals for the cubic phase, which serves as a test case. Table 8 shows results for the fundamental band 


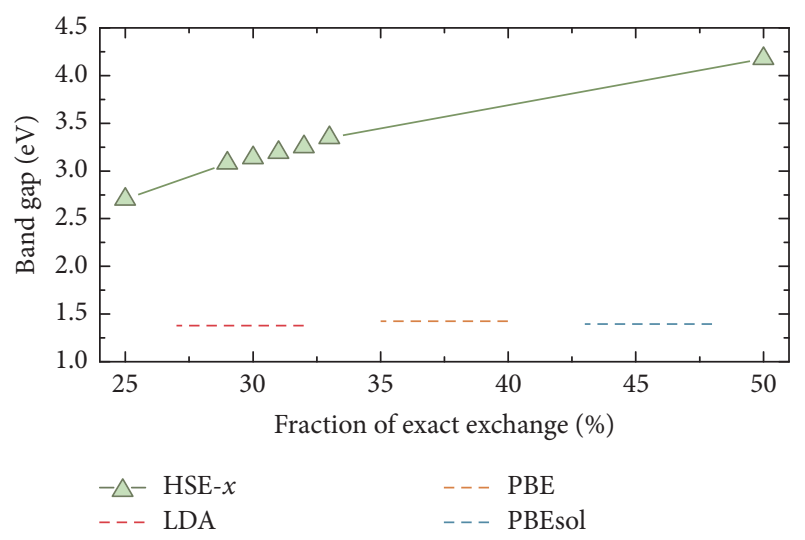

FIgURE 4: Fundamental $R_{v} \rightarrow \Gamma_{c}$ band gaps for the cubic phase of $\mathrm{KNbO}_{3}$ calculated with different exchange-correlation functionals (see Table 8). The HSE- $x$ band gaps are calculated for PBEsol equilibrium geometries and depend on the fraction of exact exchange $x$. The solid lines are intended to guide the eyes. The dashed lines mark the size of the fundamental LDA, PBE, and PBEsol band gaps.

gaps with different combinations of structural parameters (see Table 1) and functionals. In all cases, the band gap is predicted to be indirect and corresponds to a transition between the valence-band maximum at $R$, the corner of the cubic Brillouin zone, and the conduction-band minimum at $\Gamma$, its center. Furthermore, we find that the band gap is not very sensitive to the lattice parameter and that, as expected, all (semi)local functionals like LDA, PBE, or PBEsol systematically underestimate the reported experimental values, which lie between $3.14 \mathrm{eV}$ [50] and $3.24 \mathrm{eV}$ [52]. According to the preceding section, we consistently use structural parameters from PBEsol (see Table 7) from now on, and we perform a series of additional band-structure calculations with the nonlocal HSE functional. In our notation, HSE- $x$ means that the HSE functional is used with $x$ percent exact exchange, which we treat as a tunable parameter. Depending on $x$, the hybrid-functional band gap can be positioned between the lower limit of (semi)local DFT and the upper limit of HartreeFock theory. In Figure 4, the dependence of the fundamental band gap of cubic $\mathrm{KNbO}_{3}$ on the fraction of exact exchange is illustrated for $25 \leq x \leq 50$. In the most relevant region $28 \leq x \leq 32$, close to the experimentally reported band gap, an almost linear dependence on $x$ is observed. The LDA, PBE, and PBEsol band gaps are shown for comparison and exhibit an underestimation by a factor of one half. According to Table 8 , a choice of $30 \%$ exact exchange leads to much better agreement with the experimental band gap than the standard setting of $25 \%$, so that we use the HSE-30 variant in the following. The improved description of band gaps and the electronic structure in general with an increased fraction of exact exchange in HSE-type hybrid functionals has previously been demonstrated for oxide semiconductors [61] and nitride semiconductor alloys [66,67], for instance. Wang et al. [68] also noted that an increased fraction of exact exchange improves the band gap of $\mathrm{KNbO}_{3}$. Although adjusting the fraction of exact exchange for a single polymorph might not yield perfect agreement between experiment and theory for
TABLE 8: Fundamental $R_{v} \rightarrow \Gamma_{c}$ band gaps for the cubic phase of $\mathrm{KNbO}_{3}$ calculated with different lattice parameters and exchangecorrelation functionals (see Figure 4).

\begin{tabular}{lcc}
\hline Geometry & Functional & Band gap $(\mathrm{eV})$ \\
\hline Expt. & PBE & 1.426 \\
\hline LDA & LDA & 1.377 \\
\hline \multirow{2}{*}{ PBE } & PBE & 1.426 \\
& PBEsol & 1.125 \\
\hline \multirow{2}{*}{ PBEsol } & PBE & 1.540 \\
& PBEsol & 1.396 \\
\hline \multirow{4}{*}{ PBEsol } & HSE-25 & 2.701 \\
& HSE-29 & 3.080 \\
& HSE-30 & 3.138 \\
& HSE-31 & 3.194 \\
& HSE-32 & 3.252 \\
& HSE-33 & 3.346 \\
& HSE-50 & 4.176 \\
\hline
\end{tabular}

TABLE 9: Fundamental band gaps for the different phases of $\mathrm{KNbO}_{3}$ with the HSE-30 functional from the band structures in Figure 5 compared to experimental values.

\begin{tabular}{|c|c|c|c|}
\hline \multirow{2}{*}{ Phase } & \multicolumn{2}{|c|}{ HSE-30 } & \multirow{2}{*}{$\begin{array}{c}\text { Expt. } \\
\text { Band gap }(\mathrm{eV})\end{array}$} \\
\hline & Transition & Band gap $(\mathrm{eV})$ & \\
\hline$P m \overline{3} m$ & $R_{v} \rightarrow \Gamma_{c}$ & 3.14 & $3.14^{\mathrm{a}}, 3.16^{\mathrm{b}}, 3.24^{\mathrm{c}}$ \\
\hline$P 4 m m$ & $M_{v} \rightarrow \Gamma_{c}$ & 3.23 & $3.08^{\mathrm{c}}, 3.30^{\mathrm{d}}$ \\
\hline$A m m 2$ & $T_{v} \rightarrow \Gamma_{c}$ & 3.59 & $3.15^{\mathrm{c}}, 3.17^{\mathrm{e}}, 3.25^{\mathrm{f}}$ \\
\hline$P m$ & $A_{v} \rightarrow \Gamma_{c}$ & 3.63 & $3.09^{\mathrm{e}}, 3.15^{\mathrm{f}}$ \\
\hline$R 3 m$ & $Z_{v} \rightarrow \Gamma_{c}$ & 3.80 & \\
\hline
\end{tabular}

${ }^{a}$ Ref. [50]; optical gap from absorption spectrum.

${ }^{\mathrm{b}}$ Ref. [51]; optical gap from UV-vis diffuse reflectance spectrum.

${ }^{\mathrm{c}}$ Ref. [52]; optical gap from absorption spectrum.

${ }^{\mathrm{d}}$ Ref. [53]; optical gap from X-ray photoelectron spectrum.

${ }^{\mathrm{e}}$ Ref. [47]; optical gap from absorption spectrum.

${ }^{f}$ Ref. [48]; optical gap from diffuse reflectance UV-vis spectroscopy.

other phases, it represents a reasonable approach to study physical trends among the various polymorphs.

Figure 5 shows the calculated band structures for the five different phases of $\mathrm{KNbO}_{3}$. Furthermore, in Table 9, we compare the fundamental band gaps with experimental values. The calculated HSE-30 band gaps show a broad spread of $0.66 \mathrm{eV}$ between the fundamental band gaps of cubic $(3.14 \mathrm{eV})$ and rhombohedral $(3.80 \mathrm{eV}) \mathrm{KNbO}_{3}$. It should be noted that our results refer to electronic band gaps, which can be measured, in principle, by photoemission spectroscopy. No such measurements have been reported for $\mathrm{KNbO}_{3}$ so far, however. Instead, the experimental values listed in the table are deduced from optical spectroscopies and thus refer to optical band gaps. The difference between the two equals the exciton binding energy, whose magnitude is unknown for $\mathrm{KNbO}_{3}$, but detailed investigations of the related material $\mathrm{LiNbO}_{3}[25]$ suggest that it may be significant. In addition, the experimental values include thermal effects due to thermal expansion and electron-phonon coupling, particularly in the high-temperature phases, as well as size and geometry 

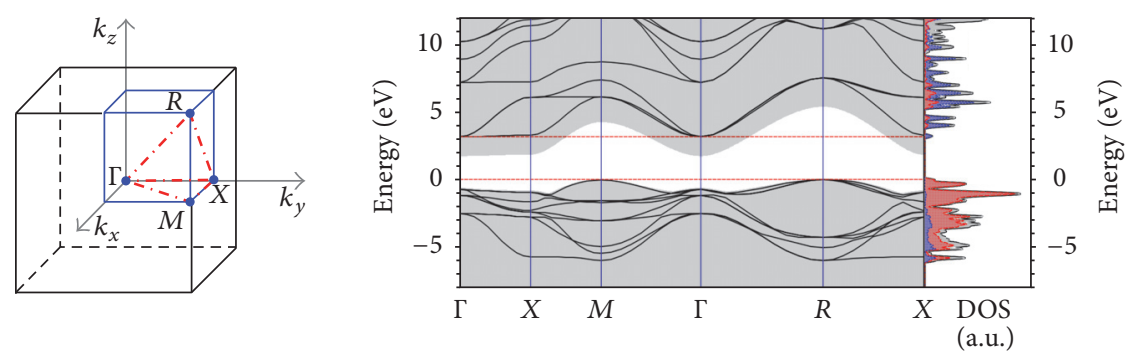

(a)
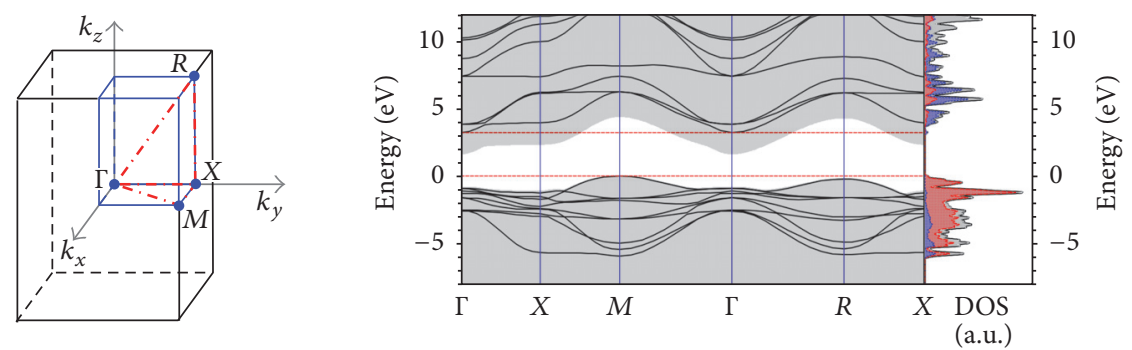

(b)
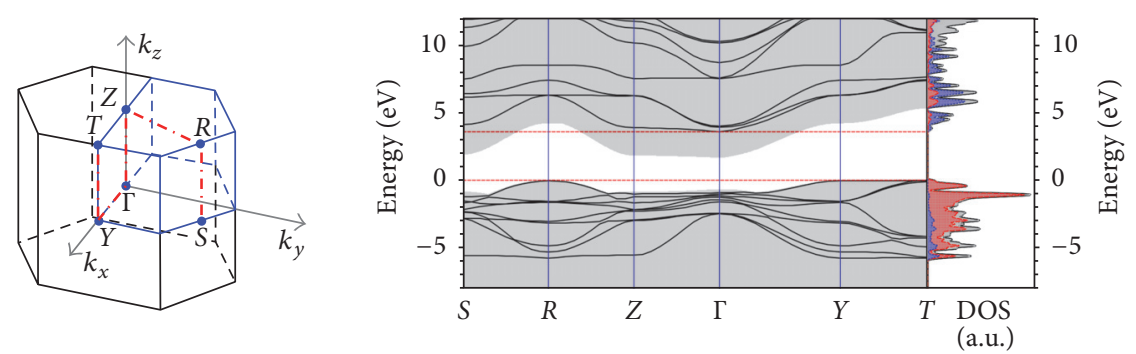

(c)
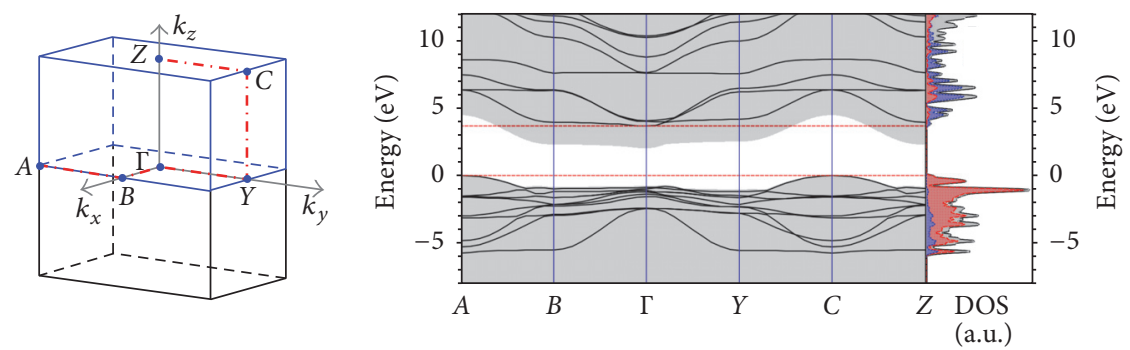

(d)
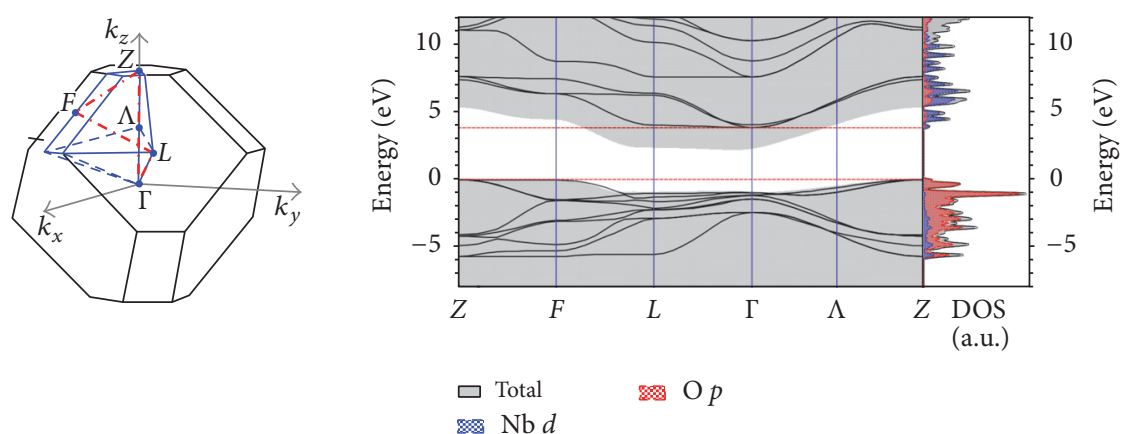

(e)

FIGURE 5: Band structures calculated with the HSE-30 functional for the cubic (a), tetragonal (b), orthorhombic (c), monoclinic (d), and rhombohedral (e) phases of $\mathrm{KNbO}_{3}$ with corresponding densities of states (DOS) in arbitrary units. The grey-shaded areas indicate the PBE band structures for comparison, and the dashed horizontal lines mark the valence-band maxima and conduction-band minima. All bandstructure calculations are based on structural data from PBEsol (see Table 7). On the left-hand side, the chosen paths within the first Brillouin zone are indicated by thick dash-dotted lines. 
TABLE 10: Calculated valence-band edge $E_{v}(\mathbf{k})$ and conduction-band edge $E_{\mathrm{c}}(\mathbf{k})$ at high-symmetry $\mathbf{k}$ points for the different phases of $\mathrm{KNbO}_{3}$. Energies are given in $\mathrm{eV}$ relative to the position of the valence-band maximum. The valence-band maximum and the conduction-band minimum are italicized.

\begin{tabular}{ccccccccccccccc}
\hline & $\begin{array}{c}\text { Cubic } \\
(P m \overline{3} m)\end{array}$ & & \multicolumn{3}{c}{$\begin{array}{c}\text { Tetragonal } \\
(P 4 m m)\end{array}$} & \multicolumn{3}{c}{$\begin{array}{c}\text { Orthorhombic } \\
(\text { Amm })\end{array}$} & \multicolumn{3}{c}{$\begin{array}{c}\text { Monoclinic } \\
(P m)\end{array}$} & \multicolumn{3}{c}{$\begin{array}{c}\text { Rhombohedral } \\
(R 3 m)\end{array}$} \\
$\mathbf{k}$ & $E_{v}(\mathbf{k})$ & $E_{c}(\mathbf{k})$ & $\mathbf{k}$ & $E_{v}(\mathbf{k})$ & $E_{c}(\mathbf{k})$ & $\mathbf{k}$ & $E_{v}(\mathbf{k})$ & $E_{c}(\mathbf{k})$ & $\mathbf{k}$ & $E_{v}(\mathbf{k})$ & $E_{c}(\mathbf{k})$ & $\mathbf{k}$ & $E_{v}(\mathbf{k})$ & $E_{c}(\mathbf{k})$ \\
\hline$\Gamma$ & -0.72 & 3.14 & $\Gamma$ & -0.90 & 3.23 & $S$ & -1.23 & 4.10 & $A$ & 0 & 6.34 & $Z$ & 0 \\
$X$ & -0.94 & 3.27 & $X$ & -1.24 & 3.96 & $R$ & -0.04 & 6.30 & $B$ & -0.97 & 3.92 & $F$ & -0.04 & 6.13 \\
$M$ & -0.04 & 6.11 & $M$ & 0 & 6.26 & $Z$ & -1.01 & 3.87 & $\Gamma$ & -0.88 & 3.63 & $L$ & -1.04 & 3.98 \\
$R$ & 0 & 7.51 & $R$ & -0.21 & 6.16 & $\Gamma$ & -0.92 & 3.59 & $Y$ & -1.19 & 4.14 & $\Gamma$ & -0.97 & 3.80 \\
& & & & & $Y$ & -0.04 & 6.30 & $C$ & -0.004 & 6.34 & $\Lambda$ & -0.60 & 5.71 \\
& & & & & $T$ & 0 & 7.36 & $Z$ & -0.97 & 3.90 & & \\
\hline
\end{tabular}

effects, because the experiments were typically not carried out for bulk samples but for nanostructures, such as microcubes in [52] or nanowires in [48]. Nanoparticles of $\mathrm{TiO}_{2}$, for instance, exhibit much smaller band gaps than in the bulk [69]. For all these reasons, the comparison in Table 9 can only be indicative. No experimental band gap is available for the rhombohedral phase, but our results are consistent with earlier calculations by Wang and Rappe [70] which yielded a fundamental band gap of $3.33 \mathrm{eV}$ with the HSE-25 functional and LDA structural data; our own HSE-25 calculation with structural data from PBEsol for the rhombohedral phase yields $3.38 \mathrm{eV}$.

The graphical band structures in Figure 5 as well as the numerical data in Table 10, which lists energy values of the highest valence band and the lowest conduction band at selected high-symmetry $\mathbf{k}$ points, confirm that in all phases there are states elsewhere in the Brillouin zone which are nearly degenerate with the valence-band maximum or the conduction-band minimum. For example, in the orthorhombic phase that is stable at room temperature, the valence-band edge at $Y$ lies only $0.04 \mathrm{eV}$ below the valence-band maximum, which is located at $T$. It is expected that such near degeneracies strongly influence the optical properties close to the absorption edge due to strong mixing of electronic transitions at different $\mathbf{k}$ points in the optical response. The corresponding densities of states (DOS) in Figure 5 share many similar features, independent of the particular polymorph. The DOS contributions from the $\mathrm{K}$ atoms are negligible around the band edges in all phases. The topmost valence bands are consistently formed by oxygen $2 p$ states and give rise to two prominent peaks close to the valence-band edge: the first peak arises from the valence-band maximum and the nearly degenerate electronic states at other high-symmetry $\mathbf{k}$ points, while the second peak originates from an almost degenerate continuum of states around $\Gamma$; its energetic distance from the conduction-band edge equals the direct band gap at $\Gamma$ for all $\mathrm{KNbO}_{3}$ phases. The lowest conduction bands, in contrast, are mainly formed by niobium $4 d$ states.

\section{Conclusions}

In summary, we have performed a comprehensive analysis of the structural and electronic properties of $\mathrm{KNbO}_{3}$ in its five known phases, including the only very recently discovered monoclinic phase, based on density-functional theory. As the energetic separation and the structural differences between the phases are small and thus potentially sensitive to details of the computational procedure, we have placed special emphasis on the influence of different approximations for the exchange-correlation functional. To this end, we compared the LDA with a range of GGAs, a meta-GGA, and, in the case of band structures, a HSE-type hybrid functional. As an important result, we find that all functionals predict the same energetic ordering of the phases, which, moreover, coincides with the order in which the phases become stable for increasing temperature. Our results further confirm that the monoclinic configuration, which has so far only been observed in synthesized nanostructures, is also metastable in the bulk. Among the considered functionals, PBEsol as well as AM05 and RTPSS yield structural data in consistently good quantitative agreement with experimental measurements for all phases; in contrast, LDA and PBE systematically underestimate and overestimate the lattice parameters, respectively, and are also less accurate with respect to the atomic displacements inside the unit cell. For this reason, we even-handedly recommend PBEsol, AM05, or RTPSS as reasonable starting points for $a b$ initio investigations with higher-level perturbative methods that require accurate equilibrium geometries. In particular, phonons, which not only are crucial in the context of phase transitions but also play a significant role for the temperature dependence of the electronic and optical properties due to the electron-phonon coupling, are sensitive to small changes in the atomic spacing.

Our band-structure calculations performed with the HSE hybrid functional and 30\% exact exchange confirm that the fundamental band gap is indirect for all phases. The variation in the size of the gap amounts to $0.66 \mathrm{eV}$ through the successive phase transitions. In particular, in the roomtemperature orthorhombic phase, the band gap is appreciably larger than in the tetragonal phase, which is often used as a structurally simpler substitute in theoretical simulations. For all phases, our results further indicate a range of near degeneracies of band-edge states in different parts of the Brillouin zone which are expected to be relevant for the linear and nonlinear optical properties of $\mathrm{KNbO}_{3}$. More elaborate $a b$ initio band-structure calculations with a proper treatment of quasiparticle and thermal effects, which go beyond the present study, are highly desirable, however. 


\section{Competing Interests}

The authors declare that there are no competing interests regarding the publication of this paper.

\section{Acknowledgments}

The authors gratefully acknowledge financial support from the Deutsche Forschungsgemeinschaft (DFG) via Sonderforschungsbereich TRR 142.

\section{References}

[1] C.-M. Fang and R. Ahuja, "Structures and stability of $\mathrm{ABO}_{3}$ orthorhombic perovskites at the Earth's mantle conditions from first-principles theory," Physics of the Earth and Planetary Interiors, vol. 157, no. 1-2, pp. 1-7, 2006.

[2] R. H. Mitchell, Perovskites: Modern and Ancient, Almaz Press, Thunder Bay, Canada, 2002.

[3] R. J. D. Tilley, Perovskites: Structure-Property Relationships, John Wiley \& Sons, Chichester, UK, 2016.

[4] B. T. Matthias, "New ferroelectric crystals," Physical Review, vol. 75, no. 11, p. 1771, 1949.

[5] S. Sanna, C. Thierfelder, S. Wippermann, T. P. Sinha, and W. G. Schmidt, "Barium titanate ground- and excited-state properties from first-principles calculations," Physical Review B, vol. 83, no. 5, Article ID 054112, 2011.

[6] A. W. Hewat, "Cubic-tetragonal-orthorhombic-rhombohedral ferroelectric transitions in perovskite potassium niobate: neutron powder profile refinement of the structures," Journal of Physics C: Solid State Physics, vol. 6, no. 16, pp. 2559-2572, 1973.

[7] S. Kim, J.-H. Lee, J. Lee et al., "Synthesis of monoclinic potassium niobate nanowires that are stable at room temperature," Journal of the American Chemical Society, vol. 135, no. 1, pp. 69, 2013.

[8] S. Simsek, H. Koc, V. A. Trepakov, A. M. Mamedov, and E. Ozbay, "Electron spectroscopy and the electronic structure of $\mathrm{KNbO}_{3}$ : first principle calculations," Ferroelectrics, vol. 461, no. 1, pp. 99-105, 2014.

[9] L. Grigorjeva, D. K. Millers, V. Pankratov et al., "Experimental and theoretical studies of polaron optical properties in $\mathrm{KNbO}_{3}$ perovskite," Solid State Communications, vol. 129, no. 11, pp. 691696, 2004.

[10] E. A. Kotomin, R. I. Eglitis, G. Borstel, L. Grigorjeva, D. Millers, and V. Pankratov, "Theoretical and experimental study of primary radiation defects in $\mathrm{KNbO}_{3}$ perovskite crystals," Nuclear Instruments and Methods in Physics Research B, vol. 166-167, pp. 299-304, 2000.

[11] E. E. Krasovskii, O. V. Krasovska, and W. Schattke, "Ab initio calculation of the optical and photoelectron spectra of $\mathrm{KNbO}_{3}$ and $\mathrm{KTaO}_{3}$," Journal of Electron Spectroscopy and Related Phenomena, vol. 83, no. 2-3, pp. 121-127, 1997.

[12] S. Torbrügge, M. Imlau, B. Schoke et al., "Optically generated small electron and hole polarons in nominally undoped and Fe-doped $\mathrm{KNbO}_{3}$ investigated by transient absorption spectroscopy," Physical Review B, vol. 78, no. 12, Article ID 125112, 2008.

[13] H. Donnerberg and M. Exner, "Derivation and application of ab initio $\mathrm{Nb}^{5+}-\mathrm{O}^{2-}$ short-range effective pair potentials in shellmodel simulations of $\mathrm{KNbO}_{3}$ and $\mathrm{KTaO}_{3}$," Physical Review B, vol. 49, no. 6, pp. 3746-3754, 1994.
[14] T. Neumann, G. Borstel, C. Scharfschwerdt, and M. Neumann, "Electronic structure of $\mathrm{KNbO}_{3}$ and $\mathrm{KTaO}_{3}$ " Physical Review B, vol. 46, no. 17, pp. 10623-10628, 1992.

[15] S. Cabuk, "Electronic structure and optical properties of $\mathrm{KNbO}_{3}$ : first principles study," Optoelectronics and Advanced Materials-Rapid Communications, vol. 1, no. 3, pp. 100-107, 2007.

[16] A. V. Postnikov, T. Neumann, G. Borstel, and M. Methfessel, "Ferroelectric structure of $\mathrm{KNbO}_{3}$ and $\mathrm{KTaO}_{3}$ from firstprinciples calculations," Physical Review B, vol. 48, no. 9, pp. 5910-5918, 1993.

[17] R. I. Eglitis, A. V. Postnikov, and G. Borstel, "Semiempirical Hartree-Fock calculations for $\mathrm{KNbO}_{3}$," Physical Review B, vol. 54, no. 4, pp. 2421-2427, 1996.

[18] E. A. Kotomin, R. I. Eglitis, A. V. Postnikov, G. Borstel, and N. E. Christensen, "First-principles and semiempirical calculations for bound-hole polarons in $\mathrm{KNbO}_{3}$," Physical Review B, vol. 60, no. 1, pp. 1-5, 1999.

[19] N. E. Christensen, E. A. Kotomin, R. I. Eglitis et al., "Quantum mechanical modelling of pure and defective $\mathrm{KNbO}_{3}$ perovskites," in Defects and Surface-Induced Effects in Advanced Perovskites, G. Borstel, A. Krumins, and D. K. Millers, Eds., pp. 3-16, Kluwer, Dordrecht, The Netherlands, 2000.

[20] P. Hohenberg and W. Kohn, "Inhomogeneous electron gas," Physical Review, vol. 136, no. 3B, pp. B864-B871, 1964.

[21] W. Kohn and L. J. Sham, "Self-consistent equations including exchange and correlation effects," Physical Review, vol. 140, no. 4A, pp. A1133-A1138, 1965.

[22] C. Thierfelder, S. Sanna, A. Schindlmayr, and W. G. Schmidt, "Do we know the band gap of lithium niobate?" Physica Status Solidi (C) Current Topics in Solid State Physics, vol. 7, no. 2, pp. 362-365, 2010.

[23] A. Riefer, S. Sanna, A. Schindlmayr, and W. G. Schmidt, "Optical response of stoichiometric and congruent lithium niobate from first-principles calculations," Physical Review B, vol. 87, no. 19, Article ID 195208, 2013.

[24] M. Friedrich, A. Riefer, S. Sanna, W. G. Schmidt, and A. Schindlmayr, "Phonon dispersion and zero-point renormalization of $\mathrm{LiNbO}_{3}$ from density-functional perturbation theory," Journal of Physics Condensed Matter, vol. 27, no. 38, Article ID 385402, 2015.

[25] A. Riefer, M. Friedrich, S. Sanna, U. Gerstmann, A. Schindlmayr, and W. G. Schmidt, "LiNbO 3 electronic structure: manybody interactions, spin-orbit coupling, and thermal effects," Physical Review B, vol. 93, no. 7, Article ID 075205, 2016.

[26] L. J. Sham and M. Schlüter, "Density-functional theory of the energy gap," Physical Review Letters, vol. 51, no. 20, pp. 18881891, 1983.

[27] J. P. Perdew, "Density functional theory and the band gap problem," International Journal of Quantum Chemistry, vol. 28, no. 19S, pp. 497-523, 1985.

[28] G. Kresse and J. Furthmüller, "Efficiency of ab-initio total energy calculations for metals and semiconductors using a plane-wave basis set," Computational Materials Science, vol. 6, no. 1, pp. 15-50, 1996.

[29] G. Kresse and D. Joubert, "From ultrasoft pseudopotentials to the projector augmented-wave method," Physical Review B, vol. 59, no. 3, pp. 1758-1775, 1999.

[30] P. E. Blöchl, "Projector augmented-wave method," Physical Review B, vol. 50, no. 24, pp. 17953-17979, 1994. 
[31] J. P. Perdew and A. Zunger, "Self-interaction correction to density-functional approximations for many-electron systems," Physical Review B, vol. 23, no. 10, pp. 5048-5079, 1981.

[32] J. P. Perdew, K. Burke, and Y. Wang, "Generalized gradient approximation for the exchange-correlation hole of a manyelectron system," Physical Review B, vol. 54, no. 23, pp. 1653316539, 1996.

[33] J. P. Perdew, A. Ruzsinszky, G. I. Csonka et al., "Restoring the density-gradient expansion for exchange in solids and surfaces," Physical Review Letters, vol. 100, no. 13, Article ID 136406, 2008.

[34] R. Armiento and A. E. Mattsson, "Functional designed to include surface effects in self-consistent density functional theory," Physical Review B, vol. 72, no. 8, Article ID 085108, 2005.

[35] A. E. Mattsson, R. Armiento, J. Paier, G. Kresse, J. M. Wills, and T. R. Mattsson, "The AM05 density functional applied to solids," Journal of Chemical Physics, vol. 128, no. 8, Article ID 084714, 2008.

[36] J. Sun, M. Marsman, G. I. Csonka et al., "Self-consistent metageneralized gradient approximation within the projector-augmented-wave method," Physical Review B, vol. 84, no. 3, Article ID 035117, 2011.

[37] J. Heyd, G. E. Scuseria, and M. Ernzerhof, "Hybrid functionals based on a screened Coulomb potential," Journal of Chemical Physics, vol. 118, no. 18, pp. 8207-8215, 2003.

[38] J. Heyd, G. E. Scuseria, and M. Ernzerhof, "Erratum: hybrid functionals based on a screened Coulomb potential [Journal of Chemical Physics 118, 8207 (2003)]," Journal of Chemical Physics, vol. 124, no. 21, Article ID 219906, 2006.

[39] J. Heyd and G. E. Scuseria, "Efficient hybrid density functional calculations in solids: assessment of the Heyd-ScuseriaErnzerhof screened Coulomb hybrid functional," Journal of Chemical Physics, vol. 121, no. 3, pp. 1187-1192, 2004.

[40] J. Heyd, J. E. Peralta, G. E. Scuseria, and R. L. Martin, "Energy band gaps and lattice parameters evaluated with the Heyd-Scuseria-Ernzerhof screened hybrid functional," Journal of Chemical Physics, vol. 123, no. 17, Article ID 174101, 2005.

[41] M. D. Fontana, G. Metrat, J. L. Servoin, and F. Gervais, "Infrared spectroscopy in $\mathrm{KNbO}_{3}$ through the successive ferroelectric phase transitions," Journal of Physics C: Solid State Physics, vol. 17, no. 3, pp. 483-514, 1984.

[42] G. I. Csonka, J. P. Perdew, A. Ruzsinszky et al., "Assessing the performance of recent density functionals for bulk solids," Physical Review B, vol. 79, no. 15, Article ID 155107, 2009.

[43] J. P. Perdew, A. Ruzsinszky, G. I. Csonka, L. A. Constantin, and J. Sun, "Workhorse semilocal density functional for condensed matter physics and quantum chemistry," Physical Review Letters, vol. 103, no. 2, Article ID 026403, 2009.

[44] R. Peverati and D. G. Truhlar, "Performance of the M11-L density functional for bandgaps and lattice constants of unary and binary semiconductors," Journal of Chemical Physics, vol. 136, no. 13, Article ID 134704, 2012.

[45] L. He, F. Liu, G. Hautier et al., "Accuracy of generalized gradient approximation functionals for density-functional perturbation theory calculations," Physical Review B, vol. 89, no. 6, Article ID 064305, 2014.

[46] C. Lin, Y. Zhao, and G. Yin, "Calculation of the lattice constant of solids with the use of valence electron structure parameters," Computational Materials Science, vol. 97, pp. 86-93, 2015.

[47] P. G. Kang, B. K. Yun, S. Shin et al., "Possible origin of stabilized monoclinic structure of $\mathrm{KNbO}_{3}$ nanomaterials at room temperature," Materials Science and Engineering B, vol. 210, pp. 19-23, 2016.
[48] T. Zhang, W. Lei, P. Liu et al., "Insights into the structure-photoreactivity relationships in well-defined perovskite ferroelectric $\mathrm{KNbO}_{3}$ nanowires," Chemical Science, vol. 6, no. 7, pp. 41184123, 2015.

[49] Z. Wu and R. E. Cohen, "More accurate generalized gradient approximation for solids," Physical Review B, vol. 73, no. 23, Article ID 235116, 2006.

[50] J. W. Liu, G. Chen, Z. H. Li, and Z. G. Zhang, "Hydrothermal synthesis and photocatalytic properties of $\mathrm{ATaO}_{3}$ and $\mathrm{ANbO}_{3}$ $(\mathrm{A}=\mathrm{Na}$ and $\mathrm{K})$," International Journal of Hydrogen Energy, vol. 32, no. 13, pp. 2269-2272, 2007.

[51] T. Su, H. Jiang, H. Gong, and Y. Zhai, "An alternative approach of solid-state reaction to prepare nanocrystalline $\mathrm{KNbO}_{3}$," Journal of Materials Science, vol. 45, no. 14, pp. 3778-3783, 2010.

[52] T. Zhang, K. Zhao, J. Yu et al., "Photocatalytic water splitting for hydrogen generation on cubic, orthorhombic, and tetragonal $\mathrm{KNbO}_{3}$ microcubes," Nanoscale, vol. 5, no. 18, pp. 8375-8383, 2013.

[53] Y. Shiozaki, E. Nakamura, and T. Mitsui, Ferroelectrics and Related Substances: Oxides Part 1: Perovskite-Type Oxides and $\mathrm{LiNbO}_{3}$ Family, New Series III/36A1, Landolt-Börnstein, 2001.

[54] M. Friedrich, A. Schindlmayr, W. G. Schmidt, and S. Sanna, " $\mathrm{LiTaO}_{3}$ phonon dispersion and ferroelectric transition calculated from first principles," Physica Status Solidi B, vol. 253, no. 4, pp. 683-689, 2016.

[55] J. K. Dewhurst and J. E. Lowther, "High-pressure structural phases of titanium dioxide," Physical Review B, vol. 54, no. 6, pp. R3673-R3675, 1996.

[56] A. Beltrán, L. Gracia, and J. Andrés, "Density functional theory study of the brookite surfaces and phase transitions between natural titania polymorphs," The Journal of Physical Chemistry $B$, vol. 110, no. 46, pp. 23417-23423, 2006.

[57] A. van de Walle and G. Ceder, "Correcting overbinding in localdensity-approximation calculations," Physical Review B, vol. 59, no. 23, pp. 14992-15001, 1999.

[58] P. Haas, F. Tran, and P. Blaha, "Calculation of the lattice constant of solids with semilocal functionals," Physical Review B, vol. 79, no. 8, Article ID 085104, 2009.

[59] S. Tinte, M. G. Stachiotti, C. O. Rodriguez, D. L. Novikov, and N. E. Christensen, "Applications of the generalized gradient approximation to ferroelectric perovskites," Physical Review B, vol. 58, no. 18, pp. 11959-11963, 1998.

[60] J. Muscat, V. Swamy, and N. M. Harrison, "First-principles calculations of the phase stability of $\mathrm{TiO}_{2}$," Physical Review $B$, vol. 65, no. 22, pp. 4112-4126, 2002.

[61] M. Landmann, E. Rauls, and W. G. Schmidt, "The electronic structure and optical response of rutile, anatase and brookite $\mathrm{TiO}_{2}$," Journal of Physics Condensed Matter, vol. 24, no. 19, Article ID 195503, 2012.

[62] F. Labat, P. Baranek, C. Domain, C. Minot, and C. Adamo, "Density functional theory analysis of the structural and electronic properties of $\mathrm{TiO}_{2}$ rutile and anatase polytypes: performances of different exchange-correlation functionals," Journal of Chemical Physics, vol. 126, no. 15, Article ID 154703, 2007.

[63] M. Gerosa, C. E. Bottani, L. Caramella, G. Onida, C. Di Valentin, and G. Pacchioni, "Electronic structure and phase stability of oxide semiconductors: performance of dielectricdependent hybrid functional DFT, benchmarked against $G W$ band structure calculations and experiments," Physical Review B, vol. 91, no. 15, Article ID 155201, 2015. 
[64] M. T. Curnan and J. R. Kitchin, "Investigating the energetic ordering of stable and metastable $\mathrm{TiO}_{2}$ polymorphs using $\mathrm{DFT}+U$ and hybrid functionals," Journal of Physical Chemistry C, vol. 119, no. 36, pp. 21060-21071, 2015.

[65] A. Schindlmayr, "The $G W$ approximation for the electronic selfenergy," in Many-Electron Approaches in Physics, Chemistry and Mathematics, V. Bach and L. Delle Site, Eds., vol. 29 of Mathematical Physics Studies, pp. 343-357, Springer International, Cham, Switzerland, 2014.

[66] M. Landmann, M. Feneberg, M. Röppischer et al., “Transition energies and direct-indirect band gap crossing in zinc-blende $\mathrm{Al}_{x} \mathrm{Ga}_{1-x} \mathrm{~N}$, , Physical Review B, vol. 87, no. 19, Article ID 195210, 2013.

[67] C. Mietze, M. Landmann, E. Rauls et al., "Band offsets in cubic GaN/AlN superlattices," Physical Review B, vol. 83, no. 19, Article ID 195301, 2011.

[68] G. Wang, H. Chen, Y. Li, A. Kuang, H. Yuan, and G. Wu, "A hybrid density functional study on the visible light photocatalytic activity of (Mo, Cr)-N codoped $\mathrm{KNbO}_{3}$," Physical Chemistry Chemical Physics, vol. 17, no. 43, pp. 28743-28753, 2015.

[69] M. M. Khan, S. A. Ansari, D. Pradhan et al., "Band gap engineered $\mathrm{TiO}_{2}$ nanoparticles for visible light induced photoelectrochemical and photocatalytic studies," Journal of Materials Chemistry A, vol. 2, no. 3, pp. 637-644, 2014.

[70] F. Wang and A. M. Rappe, "First-principles calculation of the bulk photovoltaic effect in $\mathrm{KNbO}_{3}$ and $(\mathrm{K}, \mathrm{Ba})(\mathrm{Ni}, \mathrm{Nb}) \mathrm{O}_{3-\delta}$," Physical Review B, vol. 91, no. 16, Article ID 165124, 2015. 

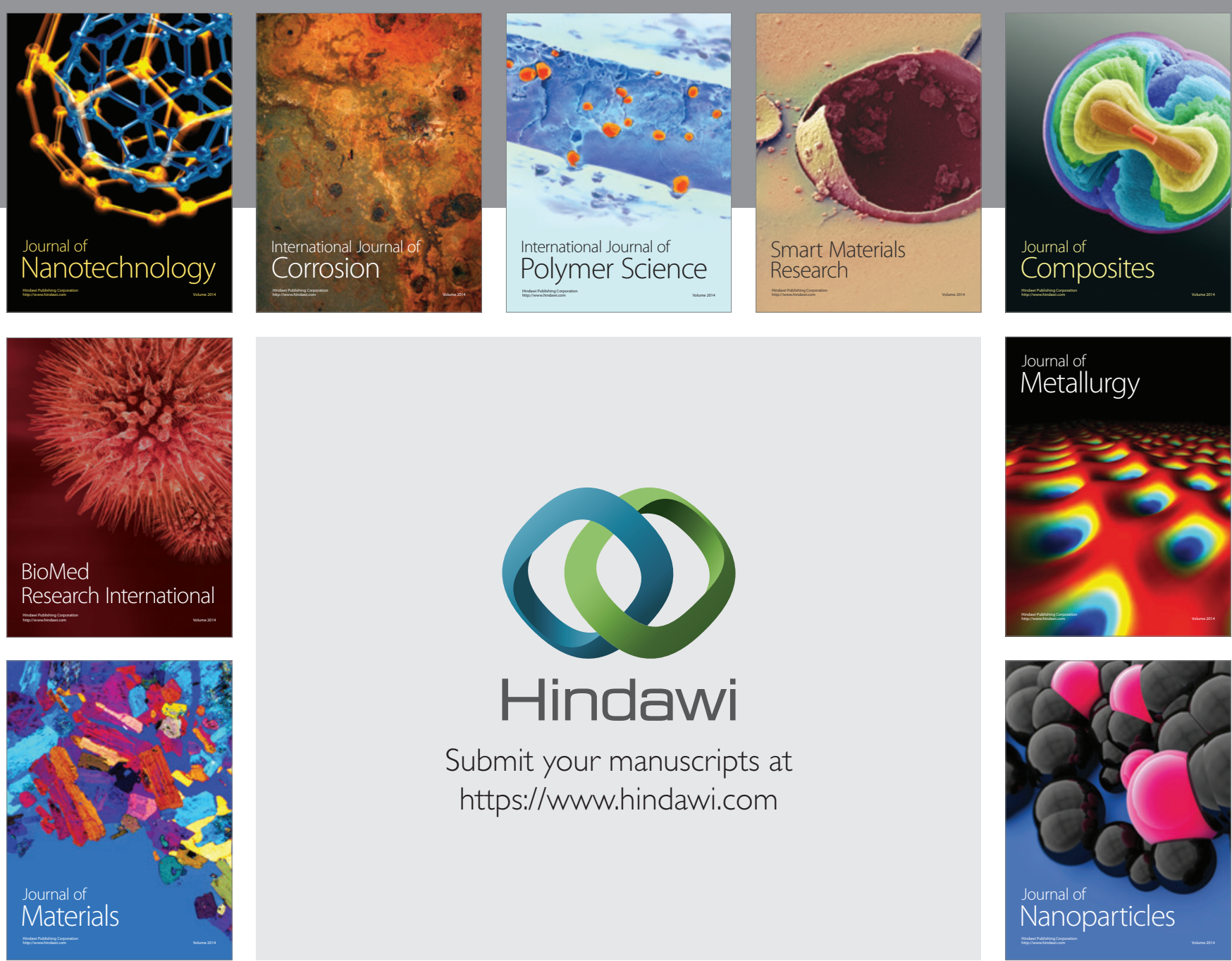

\section{Hindawi}

Submit your manuscripts at

https://www.hindawi.com

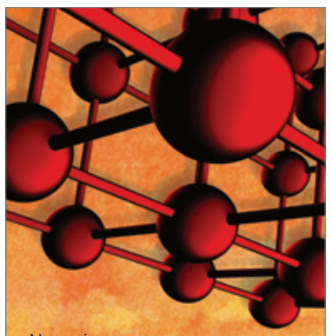

Materials Science and Engineering
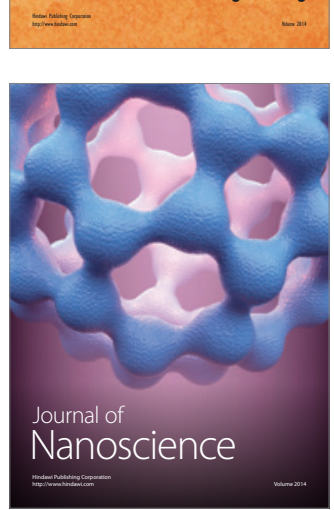
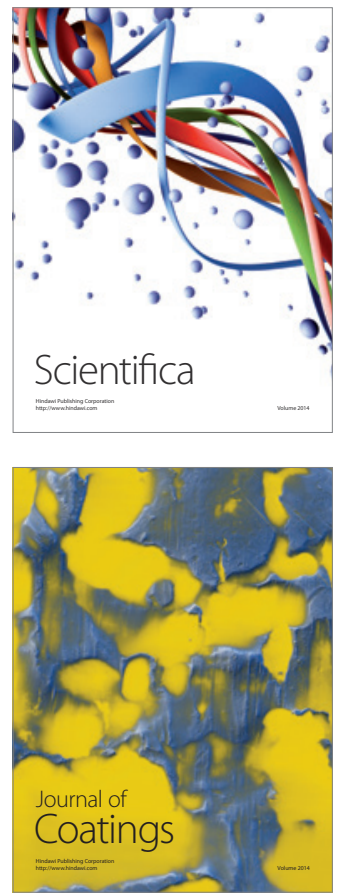
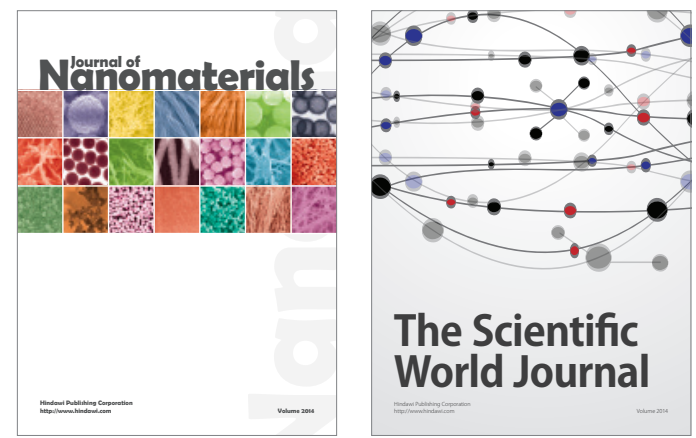

The Scientific World Journal
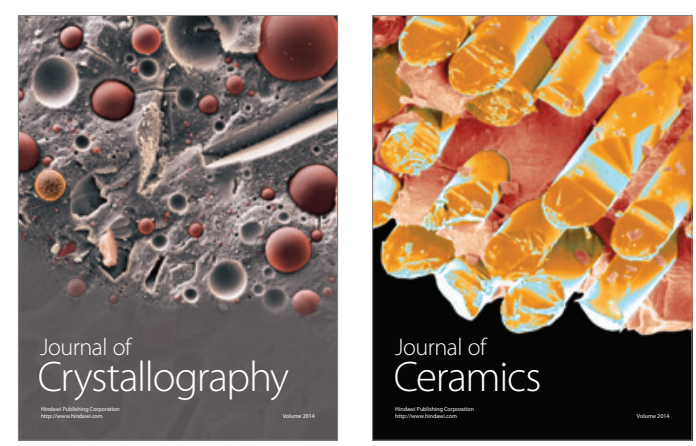
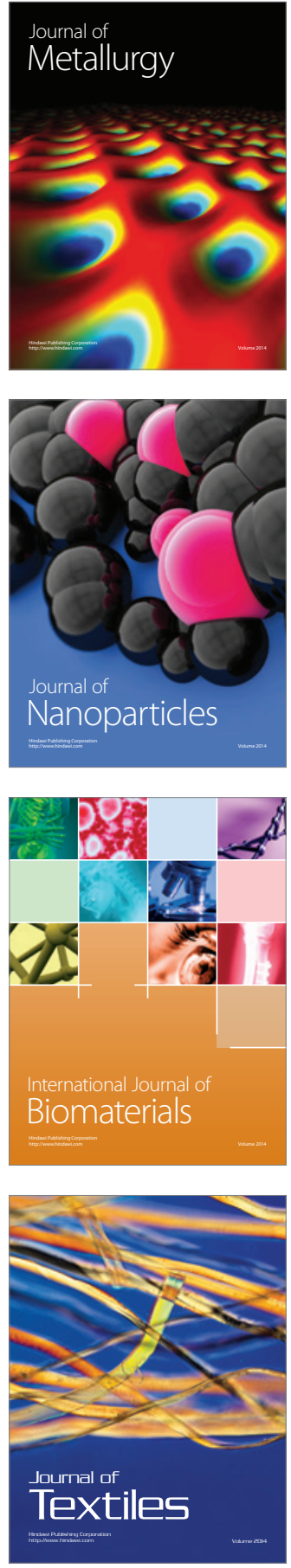